\title{
Helical waves on a vortex filament
}

\author{
Valery P. Dmitriyev \\ Lomonosov University, P. O. Box 160, Moscow 117574, Russia
}

\begin{abstract}
A small amplitude helical wave spreading along an unstretchable vortex filament in a perfect fluid obeys the linear Schrödinger equation. Taking into account elastic properties of the filament leads to the Klein-Gordon equation.
\end{abstract}

\section{Introduction}

I suggest an ideal mechanical system whose description is similar to equations that qovern the motion of a quantum particle. This system is a vortex filament in a perfect fluid.

\section{A vortex filament}

We consider a vortex tube in an ideal fluid. It can be imagined in the following way. Let a viscous fluid be pierced by a pin that spins about its axis, causing a circular motion in the fluid. The motion persists if we withdraw the pin and the fluid viscosity is removed. A very thin vortex tube will be referred to as a vortex filament.

Following the terminology customary in hydrodynamics we say that a vortex induces a velocity field in the fluid. A vortex moves with the flow of the fluid so that two straight vortex filaments rotate around each other. If we approximate small segments of a curve by straight lines, we conclude that two portions of a vortex filament will affect each other in the same way. The self-induction of a curved vortex filament causes it to evolve.

If we assume that only adjacent parts of the filament influence each other, the following law of motion of a bent vortex filament can be deduced (see, for example, [1]). The velocity $\mathbf{u}$ of the filament is proportional to the filament's curvature $\kappa$ and is directed along the binormal vector:

$$
\mathbf{u}=v \kappa \mathbf{e} \times \mathbf{n},
$$

where $v$ is a constant, the coefficient of local self-induction, that characterizes a given vortex filament, $\mathbf{n}$ is a principal normal and the tangent vector $\mathbf{e}$ is assumed to be parallel to the filament's vorticity (see Fig.1).

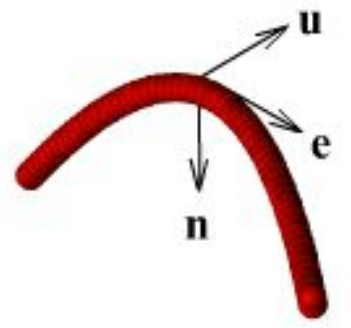

Fig.1

The drift $\mathbf{u}=\partial \mathbf{r} / \partial t$ of a bent vortex filament. Other vectors show the direction $\mathbf{e}=\partial \mathbf{r} / \partial l$ of the filament's vorticity and its curvature $\kappa \mathbf{n}=\partial^{2} \mathbf{r} / \partial l^{2}$.

In order to express Eq. (1) in Cartesian coordinates we define the spatial curve in terms of the radius vector $\mathbf{r}$ as a function of the parameter $l$; for a moving curve, there is a further dependence $\mathbf{r}(l, t)$ on time $t$ :

$$
\mathbf{r}=x(l, t) \mathbf{i}_{x}+y(l, t) \mathbf{i}_{y}+z(l, t) \mathbf{i}_{z} .
$$


Taking for $l$ the length measured along the curve from some fiducial point we may define the unit tangent vector

$$
\mathbf{e}=\frac{\partial \mathbf{r}}{\partial l}
$$

and the curvature

$$
\kappa \mathbf{n}=\frac{\partial \mathbf{e}}{\partial l} \text {. }
$$

The torsion $\tau$ of the curve is defined through the binormal $\mathbf{e} \times \mathbf{n}$ by

$$
\tau \mathbf{n}=-\frac{\partial(\mathbf{e} \times \mathbf{n})}{\partial l} \text {. }
$$

(see, for example, [2]). The velocity $\mathbf{u}$ of the liquid element of the filament is given by

$$
\mathbf{u}=\frac{\partial \mathbf{r}}{\partial t} \text {. }
$$

We are interested in the case for which the filament deviates only slightly from the rectilinear configuration. Let the filament be directed along the $x$ axis. The smallness of the deviation from the $x$ axis implies that

$$
\left|\frac{\partial y}{\partial x}\right| \ll 1, \quad\left|\frac{\partial z}{\partial x}\right| \ll 1
$$

On account of (7), the corresponding terms will be neglected in further considerations. Then we have for the arc's length

$$
d l=\left[1+\left(\frac{\partial \mathrm{y}}{\partial x}\right)^{2}+\left(\frac{\partial z}{\partial x}\right)^{2}\right]^{1 / 2} d x \approx d x
$$

and (2) is reduced to

$$
\mathbf{r}=x \mathbf{i}_{x}+y(x, t) \mathbf{i}_{y}+z(x, t) \mathbf{i}_{z} .
$$

Using (9) and (8) in (3), (4) and (6) gives

$$
\begin{gathered}
\mathbf{e}=\frac{\partial \mathbf{r}}{\partial x}=\mathbf{i}_{x}+\frac{\partial y}{\partial x} \mathbf{i}_{y}+\frac{\partial z}{\partial x} \mathbf{i}_{z} \approx \mathbf{i}_{x}, \\
\kappa \mathbf{n}=\frac{\partial \mathbf{e}}{\partial x}=\frac{\partial^{2} y}{\partial x^{2}} \mathbf{i}_{y}+\frac{\partial^{2} z}{\partial x^{2}} \mathbf{i}_{z}, \\
\mathbf{u}=\frac{\partial \mathbf{r}}{\partial t}=\frac{\partial y}{\partial t} \mathbf{i}_{y}+\frac{\partial z}{\partial t} \mathbf{i}_{z} .
\end{gathered}
$$

We see from (11) and (12) that the normal (4) and velocity (6) lie in the $y z$ plane. Substituting (10)(12) into Eq. (1) we get

$$
\frac{\partial y}{\partial t} \mathbf{i}_{y}+\frac{\partial z}{\partial t} \mathbf{i}_{z}=v \mathbf{i}_{x} \times\left(\frac{\partial^{2} y}{\partial x^{2}} \mathbf{i}_{y}+\frac{\partial^{2} z}{\partial x^{2}} \mathbf{i}_{z}\right) .
$$

According to (13), the direction of the velocity $\mathbf{u}$ can be found rotating $\mathbf{n}$ around the $x$ axis by $90^{\circ}$ from $y$ to $z$ axis. This vector algebra enables us to express Eq. (13) in a complex-valued form.

We define the complex amplitude

$$
\Phi=y+i z
$$

Equation (13) is isomorphic to

$$
\frac{\partial \Phi}{\partial t}=i v \frac{\partial^{2} \Phi}{\partial x^{2}}
$$

where the imaginary unit $i$ corresponds to the vector operator $\mathbf{i}_{x} \times$. The Frenet-Serret formulae (4) and (5) look like

$$
\kappa n=\frac{\partial^{2} \Phi}{\partial x^{2}}
$$


and

$$
\tau n=-i \frac{\partial n}{\partial x}
$$

in the complex-valued representation (14) with the complex value $n$ standing for the vector $\mathbf{n}$. In view of (16), (17) the solution to Eq. (15) is given by

$$
\Phi=a \exp [i(\tau x-\omega t)]
$$

with

$$
\omega=v \tau^{2}
$$

and

the principal normal being

$$
\kappa=a \tau^{2},
$$

$$
n=-\exp [i(\tau x-\omega t)]
$$

Using (14) and (18) in (7) we find that the Schrödinger equation (15) is valid provided that

$$
a \tau<<1 \text {, }
$$

i.e. when the curvature is much less than the torsion:

$$
\kappa<<\tau \text {. }
$$

The curve with constant curvature and torsion is referred to as a helix (see Fig.2 where the case of $\kappa>\tau$ is shown for better visulization). The helical configuration of a vortex filament rotates counter-clockwise around the $x$ axis looking in the direction of the $x$ axis with the angular velocity (19). A curvilinear configuration of an unstretchable filament can be obtained from the straight filament by adding a filament's segment to it. For a helix it amounts to $\pi a \kappa / \tau$ per a turn of the spiral.
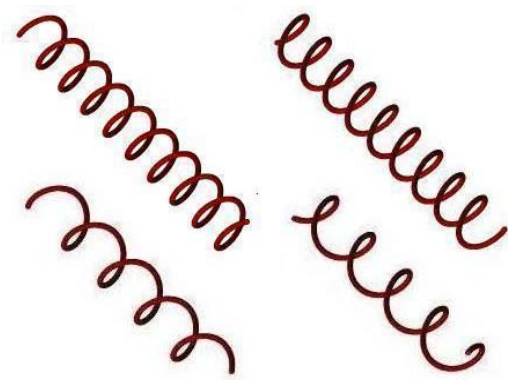

Fig.2

Left-hand screw helices (left) and right-hand screw helices (right);

$$
\kappa=4 \tau \text { (top) and } \kappa=2 \tau \text { (bottom). }
$$

We have found that for small deviations from the straight line the amplitude (14) of a vortex filament obeys the linear Schrödinger equation. Next, we may unify the description rendering it into a positionally invariant form (and thereby simplify its derivation). To this end we will differentiate Eq. (1) twice with respect to $x$ and use in the result Eqs. (6), (3) and (4) with (8) assuming that $\mathbf{e}=\mathbf{i}_{x}$. This gives the form of the motion law (1) needed

$$
\frac{\partial(\kappa \mathbf{n})}{\partial t}=v \mathbf{i}_{x} \times \frac{\partial^{2}(\kappa \mathbf{n})}{\partial x^{2}} .
$$

Rewriting Eq.(24) in complex values by the above receipt we immediately get the linear Schrödinger equation

for the complex-valued counterpart

$$
\frac{\partial \Psi}{\partial t}=i v \frac{\partial^{2} \Psi}{\partial x^{2}}
$$

$$
\Psi=\kappa n
$$

of the curvature vector $\kappa \mathbf{n}$. Substituting (21) into (26) we find the helical form complied with Eq.(25): 


$$
\Psi=-\kappa \exp [i(\tau x-\omega t)]
$$

Note that we did not presuppose constancy of $\kappa$ in the derivation of (24).

The amplitude invariant form (27) corresponds to the asymptotics, given by the condition (7), of the general solution of Eq.(1) describing a kink on the vortex filament [3]. For relatively large torsion, the nonlinear solution has the form of a spiral bulge on the filament (Fig. 3). This configuration can be loosely designated as a wave packet because of its topological similarity to the finite helical configuration obtained by the linear superposition of plain waves (simple helices) with a dispersion of wave number $\tau$. For the sake of visualization, the case $\kappa>\tau$ is shown in Fig. 3 .

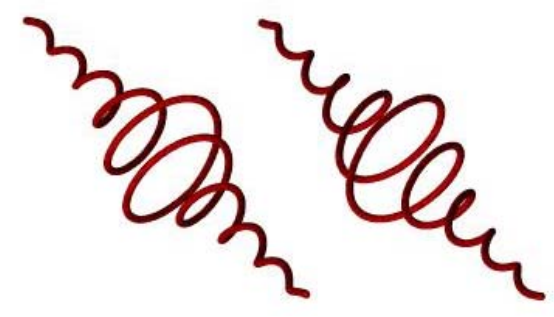

Fig.3

The wave packets comprised of left-handed (left) and right-handed (right) helices, $\kappa=10 \tau$.

Substituting (18) into the right-hand part of Eq.(15) and using in it (27) with (20) we find

$$
\frac{\partial \Phi}{\partial t}=\dot{i} \Psi
$$

By virtue of (29) we may somehow interpret the wave function $\Psi$ in physical terms as the velocity that the helix rotates about the screw axis (see for details $[4,5]$ ).

\section{An elastic vortex filament}

The elastic stretching of the vortex filament can be also taken into account. To describe it, we will first consider the motion of an elastic string.

The behavior of a rectilinear string that can be stretched elastically causing a displacement $\mathbf{s}$ in the direction of $y$ and $z$ axis is known to obey the d'Alembert equation

$$
\frac{\partial^{2} \mathbf{s}}{\partial t^{2}}=c^{2} \frac{\partial^{2} \mathbf{s}}{\partial x^{2}} \text {. }
$$

A solution to Eq. (29) is given by two independent functions:

$$
\mathbf{s}=y(x-c t) \mathbf{i}_{y}+z(x-c t) \mathbf{i}_{z} .
$$

Equation (30) specifies a hump that moves along the $x$ axis with the constant velocity $c$.

In order to describe the object that combines in itself properties of a vortex filament and of an elastic string we unite Eqs. (15) and (29) into a single equation. To this end let us rewrite Eq. (29) in a complex-valued form:

$$
\frac{\partial^{2} \Phi}{\partial t^{2}}=c^{2} \frac{\partial^{2} \Phi}{\partial x^{2}}
$$

where $\Phi$ is given by Eq. (14).

We next take advantage of the fact that the rotating helix (18) also provides a particular solution to Eq. (31). The left-hand part of Eq. (29) or (31) has the meaning of the acceleration. In order to find an addition to the acceleration due to self-induction of a vortex filament we take the second derivative of (18) with respect to time. That gives

$$
\frac{\partial^{2} \Phi}{\partial t^{2}}=-\omega^{2} \Phi .
$$


Adding the right-hand part of Eq. (32) to the right-hand part of Eq. (31) and renaming the coefficient before $\Phi$ to $\omega_{0}^{2}$ we get the equation

$$
\frac{\partial^{2} \Phi}{\partial t^{2}}=c^{2} \frac{\partial^{2} \Phi}{\partial x^{2}}-\omega_{0}^{2} \Phi
$$

which describes both the elastic stretching and self-induction of a vortex filament.

The form (18) describing a rotating helix satisfies the Klein-Gordon equation (33) with the following "relativistic" relation for the angular velocity $\omega$ of rotation

$$
\omega^{2}=\mathrm{c}^{2} \tau^{2}+\omega_{0}^{2} .
$$

In the right-hand part of Eq. (34) the term $c \tau$ corresponds to the angular velocity of rotation of an elastic string and $\omega_{0}=\nu \tau_{0}^{2}$ to that of the unstretched vortex filament.

\section{Conclusion}

Vortex filaments are structural constituents of turbulence in a fluid [6]. Perturbation waves on vortices represent a secondary formation known as soliton turbulence. A kink on a straight vortex filament evolves according to the nonlinear Schrödinger equation [3]. Schrödinger dynamics was shown to be valid not only for an isolated vortex filament, but also for perturbation waves on vortex cores in general [7]. So, the nonlinear Schrödinger equation can be used as a model of soliton turbulence $[8,9]$. In the present discourse only the case of fully thermalized soliton was considered.

We may thus conclude that the vortical turbulence provides a substratum for the deterministic Schrödinger and Klein-Gordon fields.

\section{References}

[1] G.K.Batchelor, An Introduction to Fluid Dynamics, University Press, Cambridge, 1970.

[2] R.S. Millman and G.D. Parker, Elements of Differential Geometry (Prentice-Hall, Inc., 1977).

[3] H.Hasimoto, “A soliton on a vortex filament”, J. Fluid Mech. 51 (3), 477--485 (1972).

[4] V.P.Dmitriyev, "Mechanical analogy for the wave-particle: helix on a vortex filament", J. Appl. Math. 2 (5), 241-263 (2002).

[5] V.P.Dmitriyev, "Particles and charges in the vortex sponge", Z.Naturforsch. 48a (8/9), 935-942 (1993).

[6] K.R. Sreenivasan, "Turbulence and the tube", Nature 344, No 6263, 192-193 (1990).

[7] S.Leibovich and H.Y.Ma, "Soliton propagation on vortex cores and the Hasimoto soliton", Phys. Fluids 26 (11), 3173-3179 (1983).

[8] V. E. Zakharov, A.N.Pushkarev, V.F.Shvets and V.V.Yan'kov, “About soliton turbulence”, JETP Lett. 48 (2), 83-87 (1988).

[9] R. Jordan and C.Josserand, "Self-organization in nonlinear wave turbulence", Phys. Rev. E 61 (2), 1527-1539 (2000). 